\title{
Development and validation of a nomogram risk prediction model for malignancy in dermatomyositis patients: a retrospective study
}

\author{
Jiaojiao Zhong ${ }^{\text {Equal first author, } 1,2}$, Yunan He ${ }^{\text {Equal first author, } 3}{ }^{3}$, Jianchi Ma ${ }^{1}$, Siyao Lu ${ }^{1}$, Yushi Wu ${ }^{1}$, Junmin Zhang ${ }^{\text {Corresp. } 1}$ \\ 1 Department of Dermatology, Sun Yat-sen Memorial Hospital, Sun Yat-sen University, Guangzhou, Guangdong, China \\ 2 Institute of Dermatology, Chinese Academy of Medical Sciences and Peking Union Medical College, Nanjing, China \\ 3 Reproductive Medicine Center, Tangdu Hospital, Air Force Medical University, Xi'an, China \\ Corresponding Author: Junmin Zhang \\ Email address: zhjunm@mail.sysu.edu.cn
}

Background. Dermatomyositis accompanied with malignancy is a common poor prognostic factor of dermatomyositis. Thus, the early prediction of the risk of malignancy in patients with dermatomyositis can significantly improve the prognosis of patients. However, the identification of antibodies related to malignancy in dermatomyositis patients has not been widely implemented in clinical practice. Herein, we established a predictive nomogram model for the diagnosis of dermatomyositis associated with malignancy. Methods. We retrospectively analyzed 240 cases of dermatomyositis patients admitted to Sun Yat-sen Memorial Hospital, Sun Yat-sen University from January 2002 to December 2019. According to the year of admission, the first $70 \%$ of the patients were used to establish a training cohort, and the remaining $30 \%$ were assigned to the validation cohort. Univariate analysis was performed on all variables, and statistically relevant variables were further included in a multivariate logistic regression analysis to screen for independent predictors. Finally, a nomogram was constructed based on these independent predictors. Bootstrap repeated sampling calculation C-index was used to evaluate the model's calibration, and area under the curve (AUC) was used to evaluate the model discrimination ability. Results. Multivariate logistic analysis showed that patients older than 50-year-old, dysphagia, refractory itching, and elevated creatine kinase were independent risk factors for dermatomyositis associated with malignancy, while interstitial lung disease was a protective factor. Based on this, we constructed a nomogram using the above-mentioned five factors. The $\mathrm{C}$-index was 0.780 (95\% Cl: 0.690-0.870) in the training cohort and 0.756 (95\% Cl: 0.618-0.893) in the validation cohort, while the AUC value was 0.756 (95\% Cl: 0.600-0.833). Taken together, our nomogram showed good calibration and was effective in predicting which dermatomyositis patients were at a higher risk of developing malignant tumors.

Peer) reviewing PDF | (2021:07:63440:2:1:NEW 4 Nov 2021) 
1 Development and validation of a nomogram risk prediction model for malignancy in dermatomyositis patients : a retrospective study

3 Jiaojiao Zhong ${ }^{1,2}$, Yunan $\mathrm{He}^{3}$, Jianchi $\mathrm{Ma}^{1}$, Siyao Lu ${ }^{1}$, Yushi Wu${ }^{1}$, Junmin Zhang ${ }^{1 *}$

$4{ }^{1}$ Department of Dermatology, Sun Yat-sen Memorial Hospital, Sun Yat-sen University, Guangzhou, China

52 Institute of Dermatology, Chinese Academy of Medical Sciences and Peking Union Medical College, Nanjing,

6 China

$7 \quad{ }^{3}$ Reproductive Medicine Center, Tangdu Hospital, Air Force Medical University, Xi'an, China

9 Corresponding Author:

10 Junmin Zhang, MD, PhD,

11 Department of Dermatology

Sun Yat-sen Memorial Hospital, Sun Yat-sen University

No.107 Yanjiangxi Road, 510120 Guangzhou City, Guangdong Province, China.

E-mail:junminmx@163.com

Jiaojiao Zhong and Yunan He contributed equally to the work. 


\section{Abstract}

Background. Dermatomyositis accompanied with malignancy is a common poor prognostic factor of dermatomyositis. Thus, the early prediction of the risk of malignancy in patients with dermatomyositis can significantly improve the prognosis of patients. However, the identification of antibodies related to malignancy in dermatomyositis patients has not been widely implemented in clinical practice. Herein, we established a predictive nomogram model for the diagnosis of dermatomyositis associated with malignancy.

Methods. We retrospectively analyzed 240 cases of dermatomyositis patients admitted to Sun Yat-sen Memorial

Hospital, Sun Yat-sen University from January 2002 to December 2019. According to the year of admission, the first

$70 \%$ of the patients were used to establish a training cohort, and the remaining $30 \%$ were assigned to the validation cohort. Univariate analysis was performed on all variables, and statistically relevant variables were further included in a multivariate logistic regression analysis to screen for independent predictors. Finally, a nomogram was constructed based on these independent predictors. Bootstrap repeated sampling calculation C-index was used to evaluate the model's calibration, and area under the curve (AUC) was used to evaluate the model discrimination ability.

Results. Multivariate logistic analysis showed that patients older than 50-year-old, dysphagia, refractory itching, and elevated creatine kinase were independent risk factors for dermatomyositis associated with malignancy, while interstitial lung disease was a protective factor. Based on this, we constructed a nomogram using the above-mentioned five factors. The C-index was 0.780 (95\% CI: 0.690-0.870) in the training cohort and 0.756 (95\% CI: $0.618-0.893)$ in the validation cohort, while the AUC value was 0.756 (95\% CI: 0.600-0.833). Taken together, our nomogram showed good calibration and was effective in predicting which dermatomyositis patients were at a higher risk of developing malignant tumors.

37 Keywords Dermatomyositis, malignancy, predictor, nomogram, risk prediction model 


\section{Introduction}

Dermatomyositis is a group of autoimmune diseases that mainly damage the skin and muscles and is often accompanied by tumors and internal organ damage. Skin lesions are characterized by periorbital erythema, Gottron's sign, poikiloderma and V-neck sign. Myositis often manifests as proximal muscle weakness, joint pain and dysphagia.

The relationship between dermatomyositis and malignancy was first reported by Stetz in $1916^{[1]}$. As a paraneoplastic syndrome, tumors commonly associate with dermatomyositis, including nasopharyngeal cancer, breast cancer, thyroid cancer, ovarian cancer and lung cancer and so on. The pathogenesis is reportedly related to the immune cross-reaction between tumors and normal host tissues or the secretion of functional peptides and hormones ${ }^{[2]}$. Dermatomyositis patients with underlying malignancy have a poorer prognosis compared to those without. It has been reported that the incidence of malignancy in adults with dermatomyositis ranges from $23 \%$ to $45 \%^{[3-5]}$. The 5 -year survival rate of dermatomyositis patients with malignancy ranges from $51.8 \%$ to $74.2 \%{ }^{[4,6]}$. In addition, some studies have reported that the incidence of malignancy in dermatomyositis is highest in the first year after diagnosis ${ }^{[7]}$. Therefore, it is critical to predict the probable risk of malignancy in dermatomyositis patients early.

Recently, it has been reported that a variety of myositis-specific autoantibodies (MSAs) associated with tumors has been found in patients with dermatomyositis, including transcriptional intermediary factor 1-gamma (TIF-1 $\gamma$ ), nuclear matrix protein (NXP-2), anti-small ubiquitin-like modifier activating enzyme (SAE), anti-3-hydroxy-3methylglutaryl-coenzyme A reductase (HMGCR), etc ${ }^{[8]}$. Among them, TIF $1 \gamma$ has the highest positive rate in dermatomyositis patients with underlying malignancy ${ }^{[9]}$, with about $60 \%$ to $80 \%$ of positive TIF-1 patients harboring a malignancy ${ }^{[8]}$. Although TIF $1 \gamma$ shows a strong connection between malignancy and myositis, many hospitals have not carried out TIF- $\gamma 1$ testing clinically due to the high cost. In addition, a previous meta-analysis ${ }^{[10]}$ has shown that male dermatomyositis patients older than 45-year-old with dysphagia, cutaneous necrosis, cutaneous vasculitis, rapid 
onset of myositis ( $<4$ weeks), elevated creatine kinase (CK), elevated erythrocyte sedimentation rate (ESR), and elevated C-reactive protein (CRP) were more likely to develop malignancy, while interstitial lung disease (ILD), arthritis, Raynaud syndrome and the presence of anti-Jo-1 decreased that risk. However, to date, no quantitative predictive model based on the above predictors has been established to help identify malignancy incidence in patients with dermatomyositis.

Therefore, to that end, we established a clinical prediction model for diagnosing dermatomyositis with underlying malignancy. Nomograms are generally used to transform complex regression equations into visual graphics, making the predictive model more readable and convenient for patient evaluation. A score is assigned according to each influencing factor's degree of contribution to the outcome of the variable. After which, the scores are added together to obtain the total score. Finally, the predicted value of the individual outcome event is calculated through the function conversion relationship between the total score and the probability of an outcome event ${ }^{[11,12]}$. Our predictive model was further verified both internally and externally to ensure good clinical reproducibility. To our knowledge, this is the first nomogram constructed to predict the probability of malignancy in dermatomyositis patients.

\section{Materials \& Methods}

\section{Patients}

Since this study was a retrospective study, all the patients included in the study had been discharged from the hospital, and the signature of written consent from the patients could not be obtained. However, to obtain the patients' consent, the researchers have contacted the patients in the study one by one by telephone and informed them of the contents of the informed consent forms. Oral consent has been obtained, and the patients have authorized us to use the data during their hospital stay in the study. The Ethics Committee of Sun Yat-sen Memorial Hospital, Sun Yat-sen University, has approved the oral consent for this study instead of the written informed consent (SYSEC-KY-KS- 
2020-038).

followed all relevant regulations in terms of data collection and storage. The clinical data of patients diagnosed with dermatomyositis at Sun Yat-sen Memorial Hospital, Sun Yat-sen University, from January 2002 to December 2019 were collected from the hospital database.

The inclusion criteria were according to the diagnosis criteria of dermatomyositis proposed by Bohan and Peter in 1975: (1) proximal symmetry of weak muscles in the extremities, with or without difficulty in swallowing and respiratory muscle weakness; (2) elevated muscle enzymes; (3) electromyography showing myogenic changes; (4) abnormal muscle biopsy; (5) characteristic cutaneous changes.

Exclusion criteria were as follows: (1) incomplete clinical data records and inadequate examinations; (2) unconfirmed diagnosis of dermatomyositis; (3) malignancy occurred before the diagnosis of dermatomyositis; (4) patients with overlapping syndrome, that is, combined other rheumatic immune diseases (such as systemic lupus erythematosus, scleroderma, polymyositis, rheumatoid arthritis, etc.).

The patients were selected according to the inclusion and exclusion criteria. After a diagnosis of dermatomyositis, they were divided into the malignancy and non-malignancy groups. Depending on the year of the first admission, patients from 2002 to 2015 were designated into the training cohort, while patients from 2016 to 2019 were assigned to the validation cohort.

\section{Data collection}

The following clinical data were collected: (1) general information of the patient: age, gender; (2) clinical manifestations involving Gottron's sign, periungual erythema, poikiloderma, refractory itching, V-neck sign, periorbital erythema, Raynaud's phenomenon, joint pain, proximal muscle weakness, and dysphagia; (3) laboratory 
102

indicators: antinuclear antibodies (ANA), anti-Jo-1 antibodies, CK, lactate dehydrogenase (LDH), carbohydrate antigen 125 (CA125), carbohydrate antigen 19-9 (CA19-9); (4) complications: malignancy, ILD, respiratory failure.

\section{Variables definition}

. Dermatomyositis patients with associated malignancy were diagnosed by pathological biopsy, including biopsy after tumor resection, endoscopic biopsy, and lymph node aspiration. ILD was diagnosed by chest X-ray or highresolution CT (characterized by an increased lung density with a frosted glass-like appearance in bilateral lower lung fields), pulmonary function testing, and lung biopsy. Respiratory failure refers to severe obstruction of pulmonary ventilation and/or ventilation function caused by various reasons, leading to hypoxia with/without carbon dioxide retention, with arterial blood gas values of $\mathrm{PaO}_{2}$ below $60 \mathrm{mmHg}$, with or without a $\mathrm{PaCO}_{2}$ value above $50 \mathrm{mmHg}$.

\section{Model Development}

According to the normal data range, the continuous variables in the predictors were converted into binary variables.

Then, SPSS ver. 22.0 (IBM Co., Armonk, NY, USA) was used to compare the composition of the basic data in the training and validation cohort, using the $\chi^{2}$ test (or Fisher's exact test) and t-test (or Mann-Whitney $U$ test) for categorical and continuous variables, respectively. Univariate logistic regression was used to evaluate the relationship between each independent variable and the occurrence of malignancy. The variables with significant differences $(P<$ $0.1)$ were further incorporated into our multivariate logistic analysis to screen for independent predictors ${ }^{[14]}$. The selected predictors were introduced into R ver. 3.1.2 (R Foundation for Statistical Computing, Vienna, Austria; http://www.r-project.org/), and a nomogram prediction model was constructed using the rms software package.

\section{Model validation}

Internal validation was performed using the bootstrap method for repeated sampling (1000 times). The calibration of the nomogram was evaluated by the Concordance index (C-index). The calibration curve was analyzed by plotting 
the predicted nomogram and the actual probability of malignancy in patients with dermatomyositis. The C-index of

the calibration curve ranged from 0.5 to 1 . The closer it is to 1 , the more accurate the model's prediction results are in accordance with the actual situation. For external validation, dermatomyositis patients from January 2016 to December 2019 were selected according to the same inclusion and exclusion criteria. The receiver operating characteristic curve (ROC) was drawn, and the area under the curve (AUC) was calculated to evaluate the model's discrimination ability.

The closer the AUC value is to 1, the better the model's discrimination is.

\section{Results}

\section{Patients characteristic}

A total of 289 patients with dermatomyositis were enrolled in this study. After excluding patients with incomplete data, malignancy occurring before dermatomyositis, and patients with other rheumatoid immune diseases, a total of 240 cases were selected for further analysis, including 93 men and 147 women. The average age of the patients was $46.99 \pm 18.17$ years. Among them, 54 cases had malignancy. The top 3 malignant tumors were nasopharyngeal cancer (37.0\%), lung cancer (16.7\%), breast cancer (13.0\%) （Supplement table 1) . All eligible patients were grouped by the year of admission, 168 patients admitted from 2002 to 2015 were selected for our training cohort, and 72 patients admitted to the hospital from 2016 to 2019 were picked for our validation cohort (Fig. 1). The ratio of the two groups was 7:3. The demographic, clinical characteristics and experimental results of the training and validation cohorts were similar (Table 1).

\section{Predictive factors for dermatomyositis patients with malignancy}

In the training cohort, univariate logistic regression was used to identify potential predictors of dermatomyositis with malignancy (Table 2). When contrasting the malignant tumor group to the non-malignant tumor group, we found that patients with dysphagia [odds ratio $(\mathrm{OR})=4.224 ; 95 \%$ confidence interval $(\mathrm{CI}): 1.932-9.233 ; \mathrm{P}<0.001$ ], male 
144

145

146

147

148

149

150

151

152

153

154

155

156

157

158

159

160

161

162

163

164

gender $(\mathrm{OR}=1.864 ; 95 \%$ CI: 0.909-3,780; $\mathrm{P}=0.084)$, poikiloderma $(\mathrm{OR}=2.241 ; 95 \%$ CI: 0.916-5.486; $\mathrm{P}=0.077)$,

elevated $\mathrm{CK}(\mathrm{OR}=3.137 ; 95 \%$ CI: 1.521-6.467; $\mathrm{P}=0.002)$, refractory itching $(\mathrm{OR}=3.267 ; 95 \% \mathrm{CI}: 1.274-8.378$;

$\mathrm{P}=0.014)$, above 50 years of age $(\mathrm{OR}=2.200 ; 95 \% \mathrm{CI}: 1.060-4.568 ; \mathrm{P}=0.034)$ and elevated $\mathrm{CA} 19-9(\mathrm{OR}=2.672 ; 95 \%$

CI: 1.034-6.902; $\mathrm{P}=0.042)$ were more likely to develop malignancies. While patients with ILD (OR=0.440; 95\% CI:

0.207-0.936; $\mathrm{P}=0.033$ ) were less likely to have malignancies.

After further multivariate analysis (Table 2), we found that the male gender, poikiloderma and elevated CA19-9

could not independently predict malignancy in dermatomyositis patients. For the remaining predictors, patients above

50 yeard old $(\mathrm{OR}=3.534 ; 95 \% \mathrm{CI}: 1.414-8.831 ; \mathrm{P}=0.007)$, refractory itching $(\mathrm{OR}=4.642 ; 95 \% \mathrm{CI}$ : 1.375-15.671;

$\mathrm{P}=0.013)$, dysphagia $(\mathrm{OR}=4.223$; 95\% CI: 1.617-11.023; $\mathrm{P}=0.003)$, and elevated $\mathrm{CK}(\mathrm{OR}=2.306$; 95\% CI: 1.003 -

5.304; $\mathrm{P}=0.049)$ were risk factors for dermatomyositis patients with malignancy. On the other hand, ILD (OR=0.367;

95\% CI: 0.147-0.913; $\mathrm{P}=0.031$ ) was a protective factor.

\section{Development and validation of the predictive nomogram}

Based on the predictive factors selected by the univariate and multivariate logistic regression, a nomogram was

constructed to predict the probability of malignancy occurrence in patients with dermatomyositis (Fig. 2). The value

of each factor can be determined based on the intersection of the vertical line drawn from the variable to the point

axis, and then the total risk score was calculated by adding all the variable points. The probability of malignant

occurrence can be read directly on the total point axis. In addition, to facilitate the calculation of the probability of

dermatomyositis with underlying malignancy, we provided the corresponding table form of the nomogram prediction

model (Supplement Table 2), which provided the risk points of each factor and the corresponding probability to the total risk points.

Peer) reviewing PDF | (2021:07:63440:2:1:NEW 4 Nov 2021) 
showed a good consistency between predictions from the nomogram and the actual observations (Fig. 3A). The performance of the nomogram was also evaluated in the validation cohort, with a C-index of 0.756 (95\% CI: 0.618 0.893) (Fig. 3B). The calibration curve also showed that the probability predicted by the nomogram was highly compatible clinically. In addition, the calculated AUC was 0.756 (95\% CI: 0.600-0.833), indicating that the model has a good discrimination ability (Fig. 4).

\section{Discussion}

Dermatomyositis with underlying malignancy has a high incidence and appears concurrently or successively.

Malignancy is one of the triggering factors for dermatomyositis, which induces autoimmune mechanisms through

MSAs. It has been determined that certain MSAs are significantly associated with tumor-related dermatomyositis, such as TIF-1 $\gamma$, NXP-2, SAE and HMGCR. Malignancy induces the expression and secretion of abnormal proteins (tumor-specific antigens), which are highly immunogenic and strongly cross-react with normal tissues. In addition, malignancy can express normal host proteins (tumor-associated antigen, TAA). Because TAAs are not specific to tumor cells, loss of tolerance to TAAs may cause the host to develop immunity against normal tissues, resulting in autoimmunity ${ }^{[15]}$. The sequence of dermatomyositis immune activation is unknown and may result from excessive complement activation ${ }^{[16]}$. Early diagnosis of malignancy significantly impacts prognosis, especially for tumor types that lack specific indicators and are difficult to diagnose early on. Dermatomyositis is considered to be a paraneoplastic syndrome, and most dermatomyositis patients have no other specific manifestations upon diagnosis. However, malignancy is not the only triggering factor for patients with dermatomyositis. Therefore, screening malignant patients with a high risk of dermatomyositis is of great significance for improving the early detection rate of tumors and improving the tumor prognosis.

To guide clinical management strategies, this study established a risk prediction model for dermatomyositis 
patients with underlying malignancy and screened out five predictors, namely age, dysphagia, refractory itching, ILD and elevated CK. Dysphagia, refractory itching, and elevated CK accounted for the higher scores in the predictive model and were significantly associated with malignancy. These symptoms and the results of laboratory tests were also related to dermatomyositis activity, indicating that immuno-inflammatory response was upregulated in vivo, and the autoimmune antibody titers were increased, leading to tumor-associated immune pathways activation. Besides, age also had a higher score in the prediction model. Almost all previous studies considered age to be a clear predictor, which is consistent with the biological behavior of tumors. Consequently, it is recommended that dermatomyositis patients over the age of 50 routinely undergo screening for tumors, including tumor-related history, physical examination, age-appropriate tumor marker screening, and whole-body imaging (CT, MRI or MRI) PET-CT) screening and even biopsy of suspicious lesions. ILD was found to be a protective factor for dermatomyositis associated with malignancy. Dermatomyositis patients with concurrent ILD had a significantly lower risk of developing malignancy, which is in line with the results of other highly credible clinical studies ${ }^{[10,17-19]}$. ILD is significantly related to the anti-MDA5 antibody (melanoma differentiation-associated gene 5, MDA5), especially aggressive ILD with a poor prognosis. Compared with typical dermatomyositis patients, the degree of IFN-related pathways activation in MDA5 positive patients was lighter, accompanied by a lower-activating state of STAT1, leading to a down-regulation of NOS2 expression in Th1 macrophages. At the same time, it was observed that MDA5 positive dermatomyositis patients specifically expressed NOS2 in sarcoplasmic muscle fibers and co-localized with the protective HP70 chaperone protein. These mechanisms suggest that ILD might play a role in tumor suppression ${ }^{20]}$. However, the current negative correlation between ILD and tumors in patients with dermatomyositis warrants further exploration.

In previous studies, a meta-analysis ${ }^{[10]}$ comprehensively evaluated the risk factors and protective factors of 
207

dermatomyositis associated with malignancy. There were several similarities and differences between the conclusions

of this article and previous ones. Herein, we reported that age, dysphagia, and refractory itching are risk predictors, in line with the previous meta-analysis's conclusion. However, gender was not considered a predictor in this study; although previous studies found the opposite. The meta-analysis had a lower OR value for gender as a risk factor $(\mathrm{OR}=1.92,95 \% \mathrm{CI}: 1.49-2.48)$, indicating that the reliability of gender as a high-risk factor was uncertain. The reliability of gender in epidemiology depends mainly on the number of cases, and thus, increasing the number of cases may change the status of the gender in the risk prediction model. ESR, CRP, WBC and other laboratory tests were not considered predictors, which might be due to the close relationship between laboratory tests levels and the patient's current inflammation and immune status that tend to have large fluctuations throughout the course of the disease. A retrospective study has shown that heliotrope rash and Gottron's sign are also risk factors for malignancy in dermatomyositis patients ${ }^{[21]}$, which might be related to positive anti-p155 antibody level. Anti-p155 positive dermatomyositis patients have an increased risk of malignancy ${ }^{[22]}$ and often have poikiloderma, Gottron's sign, heliotrope rash, and V-neck sign ${ }^{[21]}$. However, our findings show that these vasculitis-related lesions are not related to the onset of malignancy in patients with dermatomyositis, which might be due to the insufficient number of cases that cannot reflect the statistical differences of these variables.

A nomogram clinical prediction model for dermatomyositis patients with underlying malignancy was established for the first time based on 5 predictors. The prevalence of dermatomyositis is low, about 7-9/1,000,000[23], which greatly limited the number of cases recruited for investigation. However, the internal and external calibration curves showed consistency with clinical observations and had good predictive power. Our external verification did not use participants from other institutions; instead, new participant data at a later period in the same database was used. This verification method has been used before and conforms to the norm ${ }^{[24]}$. More importantly, the predictive indicators of 
228

this model are easy to obtain and observe, which is beneficial to physicians and has great value for identifying malignancy in patients with dermatomyositis.

Nevertheless, this study has several limitations: (1) The number of cases investigated was small since it was a single-center study. (2) The south of China is in the subtropical zone, closer to the equator, and is different from other regions in terms of climate and environment. Some studies suggested that multiple environmental factors can also trigger chronic immune activation in patients with genetic susceptibilities, such as ultraviolet light and viral infections ${ }^{[25]}$. Therefore, this study might be representative of the patient population in the south of China only. (3) Cases with incomplete clinical data have been excluded leading to possible publication bias. (4) The median followup length of patients with dermatomyositis was insufficient, which might lead to incomplete data acquisition since some patients could have developed malignant tumors at a later stage of the disease. In the future, prospective studies should be carried out with long-term follow-ups of dermatomyositis patients to obtain a more accurate incidence rate of malignant tumors.

\section{Conclusions}

This study established a simple, intuitive, and practical nomogram risk prediction model for dermatomyositis patients with underlying malignancy. Following internal and external validation, the model demonstrated a good calibration and discrimination ability. Altogether, our nomogram has good clinical translational potential, possibly helping physicians identify dermatomyositis patients at higher risk of developing malignancy. This could ultimately lead to early tumor-related screening and follow-up of high-risk patients prolonging their clinical outcome considerably. However, in order to optimize the model and improve the accuracy of prediction model, it is still necessary to collect cases from multiple centers and different regions. 


\section{Acknowledgment}

249 Thanks for all patients who provided clinical data for this article.

\section{References}

251 1. G. Stertz. Polymyositis. Berl Klin Wochenschr 1916, 53: 489.

252 2. Pelosof L. C., Gerber D. E. Paraneoplastic syndromes: an approach to diagnosis and treatment. Mayo Clin Proc $2532010,85(9): 838-854$.

254 3. Maoz C. R., Langevitz P., Livneh A., Blumstein Z., Sadeh M., Bank I., Gur H., Ehrenfeld M. High incidence of malignancies in patients with dermatomyositis and polymyositis: an 11-year analysis. Semin Arthritis Rheum 1998, 27(5): 319-324.

4. Fang Y. F., Wu Y. J., Kuo C. F., Luo S. F., Yu K. H. Malignancy in dermatomyositis and polymyositis: analysis of 192 patients. Clin Rheumatol 2016, 35(8): 1977-1984.

5. Requena C., Alfaro A., Traves V., Nagore E., Llombart B., Serra C., Martorell A., Guillen C., Sanmartin O.

Paraneoplastic dermatomyositis: a study of 12 cases. Actas Dermosifiliogr 2014, 105(7): 675-682. and prognostic factors of intracranial unruptured vertebrobasilar artery dissection. Neurology 2011, 76(20): 17351741.

7. Chen D., Yuan S., Wu X., Li H., Qiu Q., Zhan Z., Ye Y., Lian F., Liang L., Xu H., Yang X. Incidence and predictive factors for malignancies with dermatomyositis: a cohort from southern China. Clin Exp Rheumatol 2014, 32(5): 615-621. 
269

of 85 patients with idiopathic inflammatory myopathy. Medicine (Baltimore) 2010, 89(1): 47-52.

9. Hida A., Yamashita T., Hosono Y., Inoue M., Kaida K., Kadoya M., Miwa Y., Yajima N., Maezawa R., Arai S., Kurasawa K., Ito K., Shimada H., Iwanami T., Sonoo M., Hatanaka Y., Murayama S., Uchibori A., Chiba A., Aizawa H., Momoo T., Nakae Y., Sakurai Y., Shiio Y., Hashida H., Yoshizawa T., Sakiyama Y., Oda A., Inoue K., Takeuchi S., Iwata N. K., Date H., Masuda N., Mikata T., Motoyoshi Y., Uesaka Y., Maeda M. H., Nakashima R., Tsuji S., Kwak S., Mimori T., Shimizu J. Anti-TIF1-gamma antibody and cancer-associated myositis: A clinicohistopathologic study. Neurology 2016, 87(3): 299-308.

10. Lu X., Yang H., Shu X., Chen F., Zhang Y., Zhang S., Peng Q., Tian X., Wang G. Factors predicting malignancy in patients with polymyositis and dermatomyostis: a systematic review and meta-analysis. PLoS One 2014, 9(4): e94128.

11. Balachandran V. P., Gonen M., Smith J. J., DeMatteo R. P. Nomograms in oncology: more than meets the eye. Lancet Oncol 2015, 16(4): e173-180.

12. Iasonos A., Schrag D., Raj G. V., Panageas K. S. How to build and interpret a nomogram for cancer prognosis. J Clin Oncol 2008, 26(8): 1364-1370.

13. Bohan A., Peter J. B. Polymyositis and dermatomyositis (first of two parts). N Engl J Med 1975, 292(7): 344347.

14. Collins G. S., Reitsma J. B., Altman D. G., Moons K. G. Transparent reporting of a multivariable prediction model for individual prognosis or diagnosis (TRIPOD): the TRIPOD statement. BMJ 2015, 350: g7594.

15. Adler B. L., Christopher-Stine L. Triggers of inflammatory myopathy: insights into pathogenesis. Discov Med 2018, 25(136): 75-83.

16. DeWane M. E., Waldman R., Lu J. Dermatomyositis: Clinical features and pathogenesis. J Am Acad Dermatol 
290

291

292

293

294

295

296

297

298

299

300

301

302

2020, 82(2): 267-281.

17. Wang J., Guo G., Chen G., Wu B., Lu L., Bao L. Meta-analysis of the association of dermatomyositis and polymyositis with cancer. Br J Dermatol 2013, 169(4): 838-847.

18. Lega J. C., Fabien N., Reynaud Q., Durieu I., Durupt S., Dutertre M., Cordier J. F., Cottin V. The clinical phenotype associated with myositis-specific and associated autoantibodies: a meta-analysis revisiting the so-called antisynthetase syndrome. Autoimmun Rev 2014, 13(9): 883-891.

19. Ikeda S., Arita M., Misaki K., Mishima S., Takaiwa T., Nishiyama A., Ito A., Furuta K., Yokoyama T., Tokioka F., Noyama M., Yoshioka H., Ishida T. Incidence and impact of interstitial lung disease and malignancy in patients with polymyositis, dermatomyositis, and clinically amyopathic dermatomyositis: a retrospective cohort study. Springerplus 2015, 4: 240.

20. Allenbach Y., Leroux G., Suarez-Calvet X., Preusse C., Gallardo E., Hervier B., Rigolet A., Hie M., Pehl D., Limal N., Hufnagl P., Zerbe N., Meyer A., Aouizerate J., Uzunhan Y., Maisonobe T., Goebel H. H., Benveniste O., Stenzel W., French Myositis Network. Dermatomyositis With or Without Anti-Melanoma Differentiation-Associated Gene 5 Antibodies: Common Interferon Signature but Distinct NOS2 Expression. Am J Pathol 2016, 186(3): 691700.

21. Targoff I. N., Mamyrova G., Trieu E. P., Perurena O., Koneru B., O'Hanlon T. P., Miller F. W., Rider L. G., Childhood Myositis Heterogeneity Study Group, International Myositis Collaborative Study Group. A novel autoantibody to a 155-kd protein is associated with dermatomyositis. Arthritis Rheum 2006, 54(11): 3682-3689.

22. Trallero-Araguas E., Rodrigo-Pendas J. A., Selva-O'Callaghan A., Martinez-Gomez X., Bosch X., LabradorHorrillo M., Grau-Junyent J. M., Vilardell-Tarres M. Usefulness of anti-p155 autoantibody for diagnosing cancerassociated dermatomyositis: a systematic review and meta-analysis. Arthritis Rheum 2012, 64(2): 523-532. 
311 23. Sunderkotter C., Nast A., Worm M., Dengler R., Dorner T., Ganter H., Hohlfeld R., Melms A., Melzer N., Rosler

312 K., Schmidt J., Sinnreich M., Walter M. C., Wanschitz J., Wiendl H. Guidelines on dermatomyositis--excerpt from

313 the interdisciplinary S2k guidelines on myositis syndromes by the German Society of Neurology. J Dtsch Dermatol

314 Ges 2016, 14(3): 321-338.

315 24. Moons K. G., Altman D. G., Reitsma J. B., Ioannidis J. P., Macaskill P., Steyerberg E. W., Vickers A. J.,

316 Ransohoff D. F., Collins G. S. Transparent Reporting of a multivariable prediction model for Individual Prognosis or

Diagnosis (TRIPOD): explanation and elaboration. Ann Intern Med 2015, 162(1): W1-73.

25. Love L. A., Weinberg C. R., McConnaughey D. R., Oddis C. V., Medsger T. A., Jr., Reveille J. D., Arnett F. C.,

Targoff I. N., Miller F. W. Ultraviolet radiation intensity predicts the relative distribution of dermatomyositis and anti-

Mi-2 autoantibodies in women. Arthritis Rheum 2009, 60(8): 2499-2504. 


\begin{tabular}{ccc}
\hline \multirow{2}{*}{ Characteristic } & \multicolumn{2}{c}{ Training cohort (n=168) } \\
\cline { 2 - 3 } & N & Percent (\%) \\
\hline
\end{tabular}

\section{Basic information}

Age (years), mean $\pm \mathrm{SD}$

$48.09 \pm 18.69$

Validation cohort $(n=72)$

Sex

\section{Male}

65

38.7

Percent (\%)

\section{$\mathbf{P}$}

N $\quad$ Percent (\%)

$\overline{\mathbf{P}}$

Female

103

61.3

$44.43 \pm 16.73$

0.153

0.977

\section{Clinical manifestation}

Gottron's sign

yes

no

Periungual erythema

yes

no

Poikiloderma

yes

no

Refractory itching

yes

no

V-neck sign

$$
\text { yes }
$$

no

Periorbital erythema

$$
\text { yes }
$$

no

Raynaud's phenomenon

$$
\text { yes }
$$

no

Joint pain
84

84

19

149

47

121

21

147

70

98

113

55

8

160
50.0

50.0

11.3

88.7

28.0

72.0

12.5

87.5

41.7

58.3

67.3

32.7

4.8

95.2
41

0.185

43

29

0.792

9

63

87.5

12.5

13.9

86.1

62

0.562

11

61

84.7

15.3

43.1

56.9

58.3

$\begin{array}{ll}30 & 41.7\end{array}$

$4 \quad 5.6$

$\begin{array}{ll}67 & 94.4\end{array}$

0.167

0.019

0.842

0.778 


$\begin{array}{lcccc}\text { yes } & 23 & 13.7 & 14 & 19.4 \\ \text { no } & 145 & 86.3 & 58 & 80.6\end{array}$

Proximal muscle weakness

0.829

yes 119

49

70.8

50

69.4

no

29.2

22

30.6

Dysphagia

0.837

yes $\quad 37$

22.0

15

20.8

no

131

78.0

57

79.2

\section{Complication}

Malignant tumor

0.157

yes

42

25.0

12

16.7

no

126

75.5

$60 \quad 83.3$

Interstitial pneumonia

yes 72

42.9

35

48.6

no

96

57.1

37

51.4

Respiration failure

30

17.9

18

0.205

yes

138

82.1

54

25.0

no

75.0

\section{Laboratory values}

CK (U/L)

69

99

$<198$

LDH (U/L)

$$
\begin{aligned}
& \geq 300 \\
& <300
\end{aligned}
$$

ANA

Positive

Negative

Anti-Jo-1

Positive

65

65

4
103

61.3

38.7

41.1

58.9

20

52

36

36

61.3

45

38.7

27
2.4
0.104

0.862

62.5

0.058

27.8

72.2

50.0

50.0

37.5

0.004

11.1 
Negative

CA125(U/ml)

$\geq 35$

$<35$

CA19-9(U/ml)

$\geq 37$

$<37$
164

97.6

15

153

21

146

91.1

12.6

87.4
64

4

68
0.375

5.6

94.4

6.9

93.1

322 CK: creatine kinase; LDH: lactate dehydrogenase; ANA: antinuclear antibody; CA125:carbohydrate antigen

323 125; CA19-9: carbohydrate antigen 19-9. 
Table 2 Univariate and multivariate analysis of risk factors in training cohort

\begin{tabular}{|c|c|c|c|c|}
\hline \multirow{2}{*}{ Factors } & \multicolumn{2}{|c|}{ Univariate } & \multicolumn{2}{|c|}{ Multivariate } \\
\hline & $\mathrm{P}$ & $\mathrm{OR}(95 \% \mathrm{CI})$ & $\mathrm{P}$ & OR $(95 \% \mathrm{CI})$ \\
\hline Age $\geq 50 y$ & $0.034 \dagger$ & $2.200(1.060,4.568)$ & $0.007 *$ & $3.534(1.414,8.831)$ \\
\hline Sex & $0.084 \dagger$ & $1.864(0.909,3.780)$ & 0.180 & $1.804(0.761,4.276)$ \\
\hline Gottron's sign & 0.286 & $0.682(0.337,1.379)$ & & \\
\hline Periochia erythema & 0.888 & $1.081(0.365,3.205)$ & & \\
\hline Poikiloderma & $0.077 \dagger$ & $2.241(0.916,5.486)$ & 0.051 & $2.890(0.994,8.407)$ \\
\hline Refractory itching & $0.014 \dagger$ & $3.267(1.274,8.378)$ & $0.013^{*}$ & $4.642(1.375,15.671)$ \\
\hline V-neck sign & 0.857 & $1.067(0.527,2.163)$ & & \\
\hline Periorbital erythema & 0.507 & $1.295(0.603,2.781)$ & & \\
\hline Raynaud's phenomenon & $>0.999$ & $<0.001$ & & \\
\hline Joint pain & 0.165 & $0.408(0.115,1.449)$ & & \\
\hline Proximal muscle weakness & 0.769 & $0.892(0.417,1.908)$ & & \\
\hline Dysphagia & $<0.001 \dagger$ & $4.224(1.932,9.233)$ & $0.003^{*}$ & $4.223(1.617,11.023)$ \\
\hline Interstitial pneumonia & $0.033 \dagger$ & $0.440(0.207,0.936)$ & $0.031 *$ & $0.367(0.147,0.913)$ \\
\hline Respiration failure & 0.250 & $0.546(0.195,1.531)$ & & \\
\hline $\mathrm{CK} \geq 198 \mathrm{U} / \mathrm{L}$ & $0.002 \dagger$ & $3.137(1.521,6.467)$ & $0.049 *$ & $2.306(1.003,5.304)$ \\
\hline $\mathrm{LDH} \geq 300 \mathrm{U} / \mathrm{L}$ & 0.784 & $0.905(0.443,1.847)$ & & \\
\hline ANA $(+)$ & 0.316 & $0.696(0.343,1.412)$ & & \\
\hline Anti-Jo-1(+) & $>0.999$ & $<0.001$ & & \\
\hline $\mathrm{CA} 125 \geq 35 \mathrm{U} / \mathrm{ml}$ & 0.876 & $1.100(0.331,3.660)$ & & \\
\hline CA19-9 $\geq 37 \mathrm{U} / \mathrm{ml}$ & $0.042 \dagger$ & $2.672(1.034,6.902)$ & 0.319 & $1.811(0.563,5.824)$ \\
\hline
\end{tabular}

OR, odds ratio; $\mathrm{CI}$, confidence interval.

$\dagger P<0.1,{ }^{*} P<0.05$, statistically significant difference.

324

325

326

327 
Figure 1

Figure 1 Flow chart for cases selection

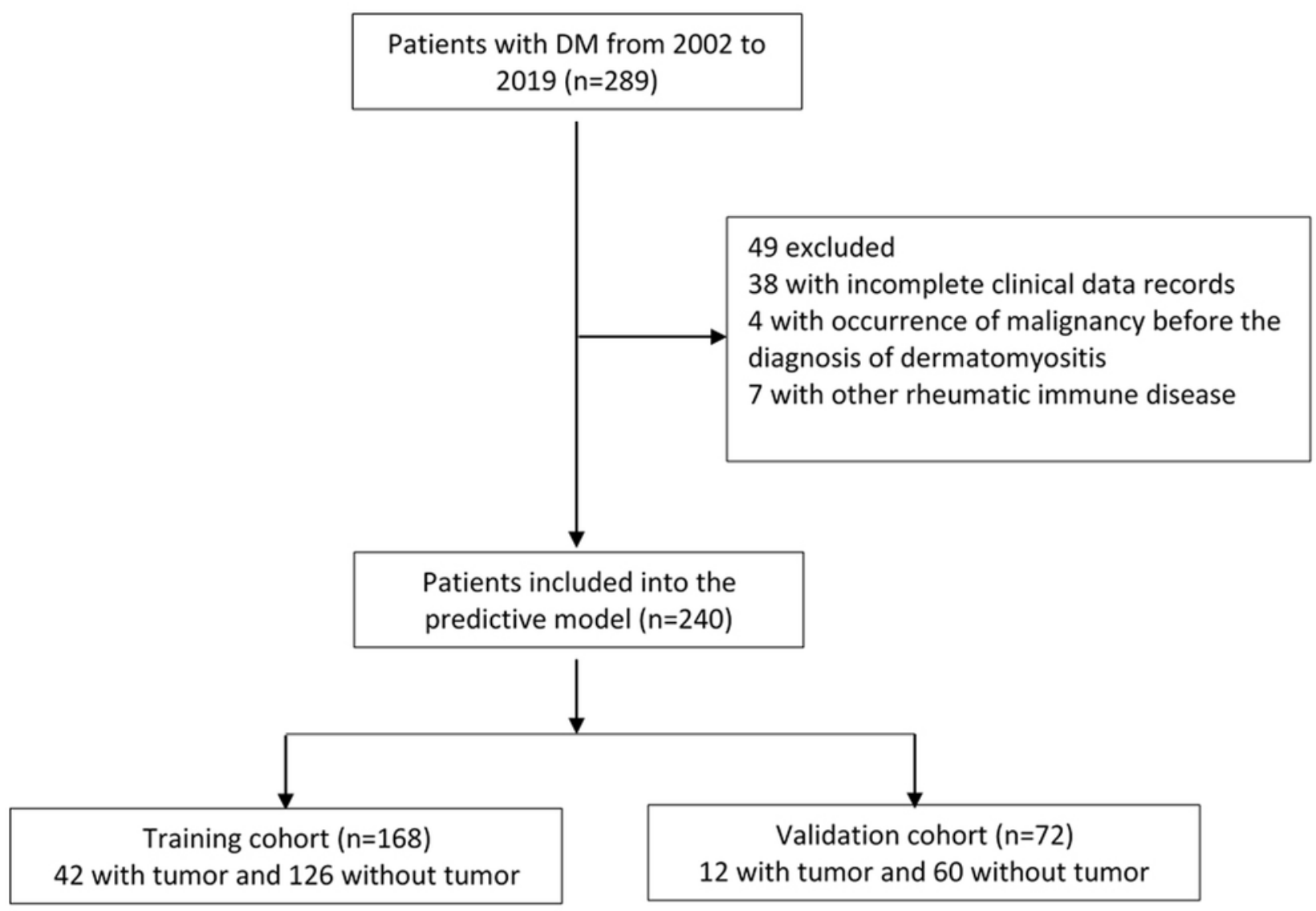


Figure 2

Figure 2 Nomogram for individualized prediction of malignancy in patients with dermatomyositis

Points

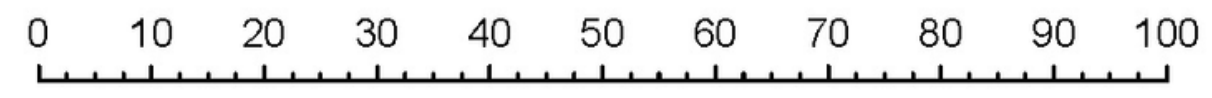

Age

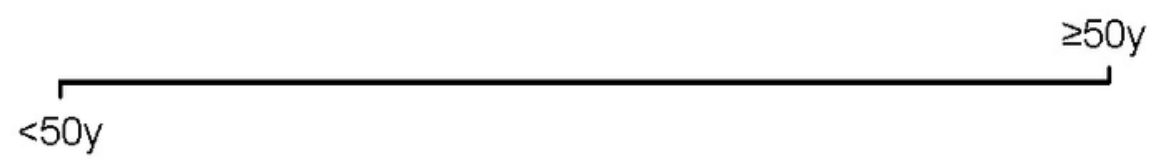

Interstitial lung

disease

no

Dysphagia
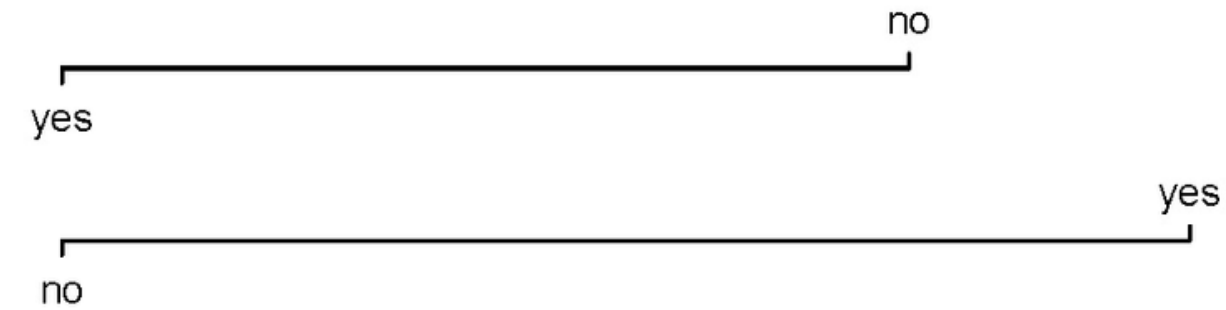

Refractory itching

no

Creatine kinase $\geq 198 \mathrm{U} / \mathrm{L}$

$<198$ U/L

Total Points

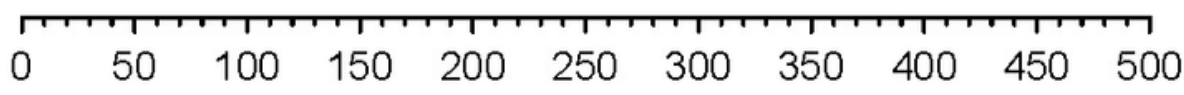

Risk

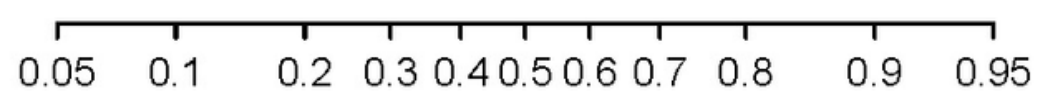


Figure 3

Figure 3 Calibration curve comparing predicted and actual probabilities of dermatomyositis with malignancy in the training cohort $(A)$ and in the validation cohort (B).

A

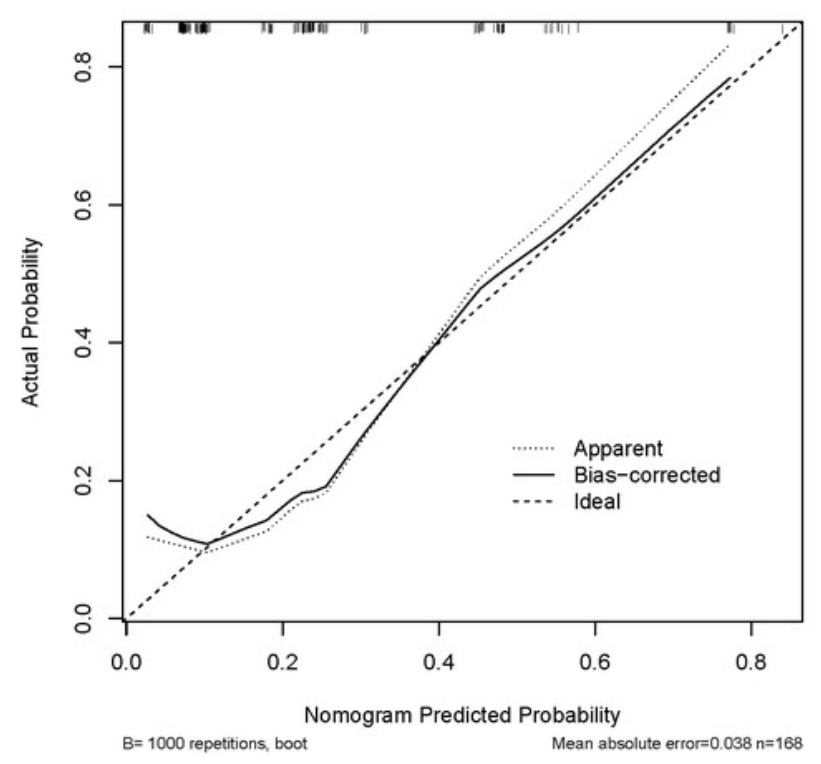

B

Calibration Curve

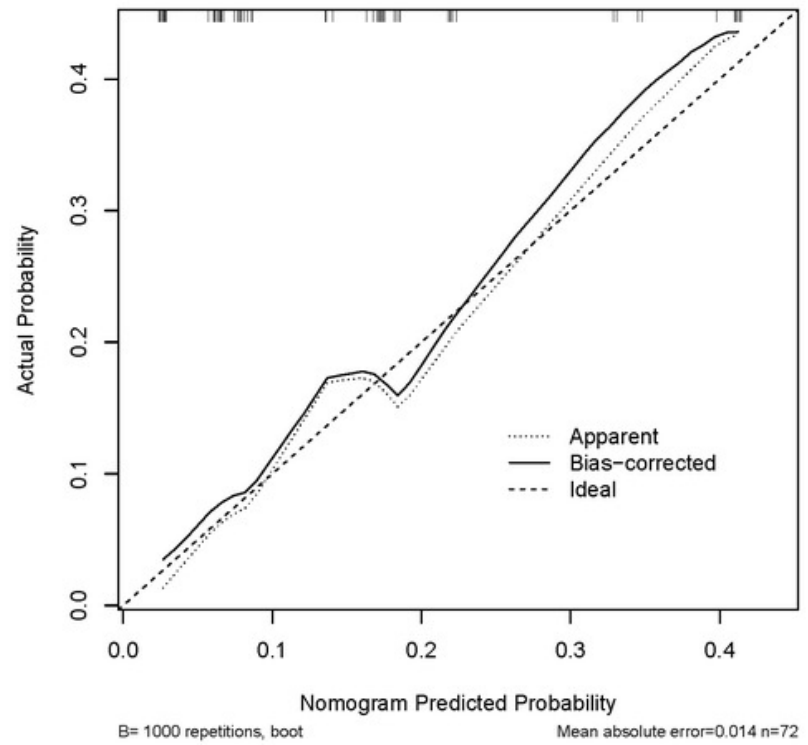


Figure 4

Figure 4 Receiver under the operator characteristic (ROC) curve for the test accuracy in the validation cohort.

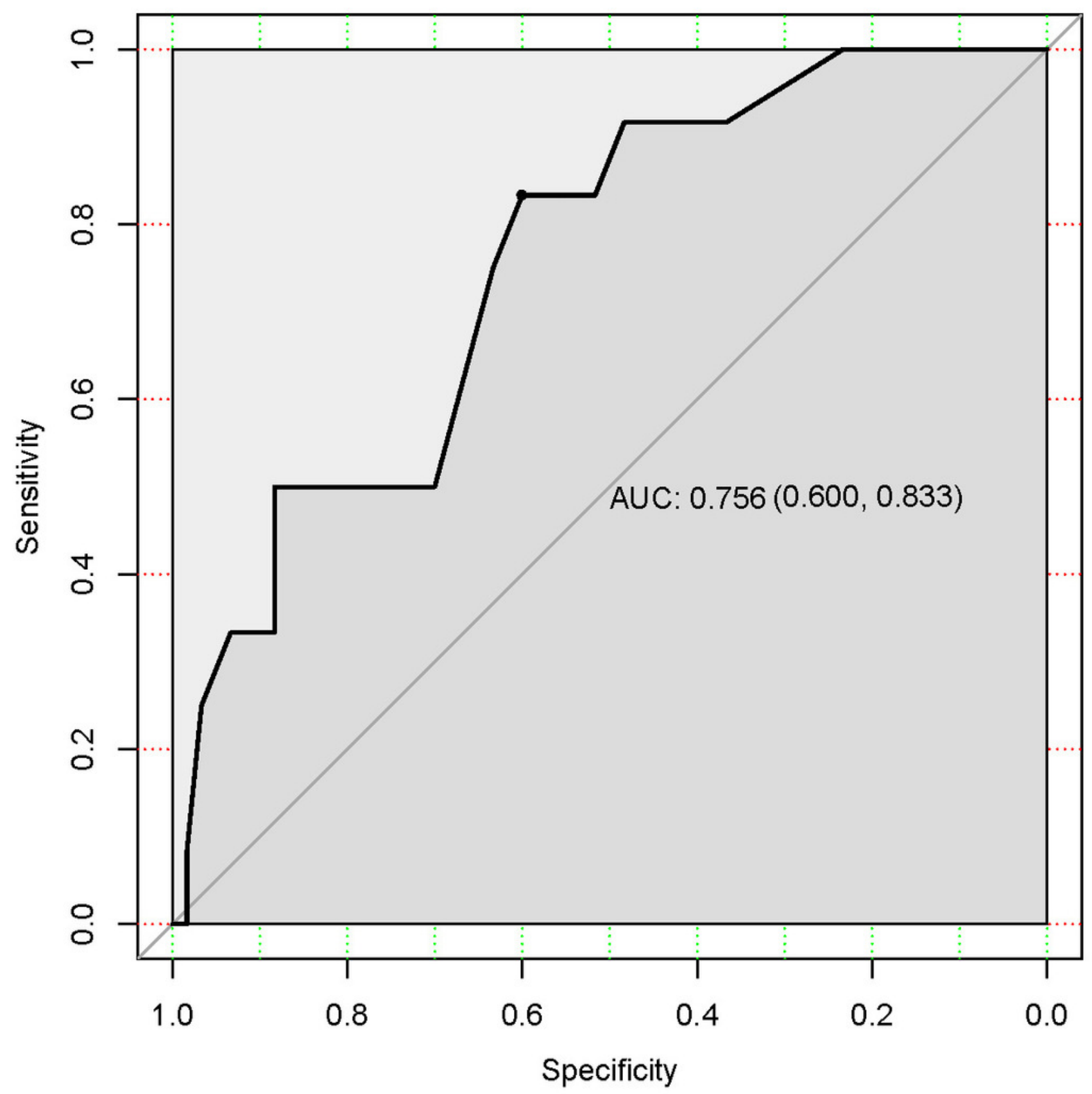

